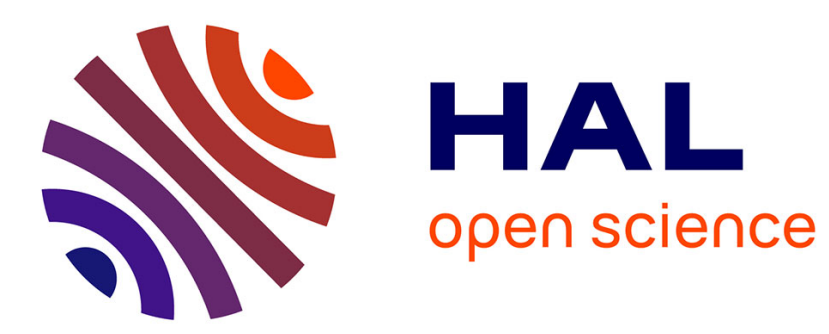

\title{
Vibrational contribution to the surface specific heat of a fluid
}

\author{
Bahram Djafari-Rouhani, L. Dobrzynski
}

\section{To cite this version:}

Bahram Djafari-Rouhani, L. Dobrzynski. Vibrational contribution to the surface specific heat of a fluid. Journal de Physique, 1982, 43 (3), pp.523-529. 10.1051/jphys:01982004303052300 . jpa00209422

\section{HAL Id: jpa-00209422 https://hal.science/jpa-00209422}

Submitted on 1 Jan 1982

HAL is a multi-disciplinary open access archive for the deposit and dissemination of scientific research documents, whether they are published or not. The documents may come from teaching and research institutions in France or abroad, or from public or private research centers.
L'archive ouverte pluridisciplinaire HAL, est destinée au dépôt et à la diffusion de documents scientifiques de niveau recherche, publiés ou non, émanant des établissements d'enseignement et de recherche français ou étrangers, des laboratoires publics ou privés. 
Classification

Physics Abstracts

$62.60-65.20$

\title{
Vibrational contribution to the surface specific heat of a fluid
}

\author{
B. Djafari-Rouhani and L. Dobrzynski \\ Laboratoire des Surfaces et Interfaces (*), I.S.E.N., 3, rue F.-Baës, 59046 Lille Cedex, France
}

(Reçu le 23 juillet 1981, accepté le 24 novembre 1981)

Résumé. - Nous donnons des résultats analytiques pour la contribution vibrationnelle à la chaleur spécifique $\Delta C_{V}(T)$ de basse température d'un liquide non visqueux. Il est montré par une méthode de fonctions de Green qu'en présence d'ondes capillaires de surface

$$
\Delta C_{V}(T)=M T^{4 / 3}+N T^{2}+P T^{8 / 3}+Q T^{3}+\mathrm{O}\left(T^{10 / 3}\right) .
$$

Les coefficients $M, N, P$ et $Q$ sont donnés analytiquement en fonction de la densité du liquide, de la tension superficielle et de la vitesse longitudinale du son dans le volume du liquide.

\begin{abstract}
We report analytical results for the vibrational contributions to the low temperature surface specific heat $\Delta C_{V}(T)$ of a non viscous liquid. It is shown by a Green's function method that when capillary waves exist on the surface of such a liquid

$$
\Delta C_{V}(T)=M T^{4 / 3}+N T^{2}+P T^{8 / 3}+Q T^{3}+\mathrm{O}\left(T^{10 / 3}\right) .
$$

The coefficients $M, N, P$ and $Q$ are given in closed form as functions of the density of the liquid, the surface tension and the velocity of the longitudinal bulk waves.
\end{abstract}

1. Introduction. - The surface contribution to the specific heat of a finite crystal has been studied extensively [1] and its low temperature $T^{2}$ behaviour is well established both theoretically and experimentally. Although Atkins [2] has shown that a $T^{4 / 3}$ contribution to the low temperature surface specific heat $\Delta C_{V}(T)$ of a liquid may be due to hydrodynamic capillary waves, he established this result by taking into account only the existence of these capillary waves, without considering the corresponding perturbation of the bulk density of states. Recently Iosilevskii [3] reported that $\Delta C_{V}(T)$ behaves like $T^{2}$ at low temperature, without any mention of the $T^{4 / 3}$ behaviour predicted by Atkins [2].

Therefore a thorough examination of this question was needed. We report here such a study that takes into account the effect of the capillary waves as well as the perturbation of the bulk waves due to the existence of the surface. We use for this purpose a Green's function method [4]. Different formulations [3, 5-8] of this method were already used for the study of other

(*) Associé au C.N.R.S. vibrational properties of liquid surfaces, namely surface modes and Brillouin scattering.

In section 2 we outline the derivation of the Green's function for a semi-infinite liquid. The fluid will be assumed compressible and viscous. The general formulation of this problem is similar to that of an isotropic solid [9]. Going then to the limit of an inviscid liquid, we give the contribution of the surface to the density of states and to the specific heat (Section 3). -This last quantity contains terms with different powers of the temperature $T$, coming from different contributions to the density of states. We give here all these terms up through the one in $T^{3}$ which has the same power as the contribution of the bulk waves to the specific heat.

2. Formulation of the Green's function. - The motion of the bulk fluid is governed by the NavierStokes equation

$$
\begin{aligned}
\rho\left[\frac{\partial \mathbf{v}}{\partial t}+\right. & (\mathbf{v} \cdot \boldsymbol{\nabla}) \mathbf{v}]= \\
& =-\nabla p+\mu \nabla^{2} \mathbf{v}+\left(\mu^{\prime}+\frac{1}{3} \mu\right) \nabla(\nabla \cdot \mathbf{v})
\end{aligned}
$$


where $\mathbf{v}$ is the velocity, $\rho$ the density, $\mu$ and $\mu^{\prime}$ the coefficients of shear and dilatation viscosity and $p$ the pressure. For small velocities, the equation (1) can be linearized by neglecting the term (v. V) v. Assuming that the fluid excitations execute harmonic motion with a time dependence $\mathrm{e}^{-i \omega t}$, the velocity $\mathbf{v}$ is related to the displacement $\mathbf{u}$ by

$$
\mathbf{v}=-i \omega \mathbf{u} \text {. }
$$

Finally, we assume that the pressure term in equation (1) is due only to the density fluctuations giving rise to dilatation and compression of the fluid. That is, we neglect the contribution due to the thermal fluctuations. Then

$$
p=-\lambda(\nabla \cdot \mathbf{u}),
$$

where $\lambda$ is the compressibility of the fluid which is related to the (longitudinal) sound velocity $C$

$$
C^{2}=\frac{\lambda}{\rho} \text {. }
$$

With the above assumptions, the stress tensor in the liquid can be written as

$$
\begin{array}{r}
\sigma_{\alpha \beta}=\lambda(\nabla . u) \delta_{\alpha \beta}+\mu\left[\frac{\partial v_{\alpha}}{\partial x_{\beta}}+\frac{\partial v_{\beta}}{\partial x_{\alpha}}-\frac{2}{3} \delta_{\alpha \beta}(\nabla \cdot v)\right]+ \\
+\mu^{\prime} \delta_{\alpha \beta}(\nabla \cdot v),
\end{array}
$$

with $\alpha, \beta=1,2,3$.

This form of the stress tensor is completely analogous to that obtained in elasticity theory for an isotropic solid. The following correspondances should be made

$$
C_{1}^{2}=C^{2}-\frac{i \omega}{\rho}\left(\mu^{\prime}+\frac{4}{3} \mu\right), \quad C_{\mathrm{t}}^{2}=-\frac{i \omega \mu}{\rho},
$$

where $C_{1}$ and $C_{\mathrm{t}}$ are the velocities of longitudinal and transverse sound waves in the solid.

The derivation of the bulk Green's function can be done step by step along the same lines as for an isotropic solid [9]. Let us recall the equation satisfied by this Green's function, after making a Fourier analysis parallel to the plane of the surface (plane $x_{3}=0$ ) and a rotation of the $x_{1}, x_{2}$ axes which brings the $x_{1}$ axis along the wavevector $\mathbf{k}_{\|}$parallel to the surface

$$
\left(\begin{array}{ccc}
\omega^{2}-\frac{k_{\|}^{2}}{\rho}\left[\lambda-i \omega\left(\mu^{\prime}+\frac{4}{3} \mu\right)\right]-\frac{i \omega \mu}{\rho} \frac{\mathrm{d}^{2}}{\mathrm{~d} x_{3}^{2}} & 0 & \frac{i k_{\|}}{\rho}\left[\lambda-i \omega\left(\mu^{\prime}+\frac{1}{3} \mu\right)\right] \frac{\mathrm{d}}{\mathrm{d} x_{3}} \\
0 & \omega^{2}-\frac{i \omega \mu}{\rho}\left(\frac{\mathrm{d}^{2}}{\mathrm{~d} x_{3}^{2}}-k_{\|}^{2}\right) & 0 \\
\frac{i k_{\|}}{\rho}\left[\lambda-i \omega\left(\mu^{\prime}+\frac{1}{3} \mu\right)\right] \frac{\mathrm{d}}{\mathrm{d} x_{3}} & 0 & \omega^{2}+\frac{1}{\rho}\left[\lambda-i \omega\left(\mu^{\prime}+\frac{4}{3} \mu\right)\right] \frac{\mathrm{d}^{2}}{\mathrm{~d} x_{3}^{2}}+\frac{i \omega \mu}{\rho} k_{\|}^{2}
\end{array}\right) \times
$$

where $g_{i j}$ stands for $g_{i j}\left(k_{\|} \omega^{2} \mid x_{3}, x_{3}^{\prime}\right)$. Let us also recall that equation (7) leads to $g_{12}=g_{21}=g_{23}=g_{32}=0$, that is to a decoupling between sagittal and transverse polarization. The solution of equation (7) for the bulk fluid is exactly the same as for an isotropic solid [9], when we make use of the relations (6). In the presence of a free surface, equation (7) has to be solved subject to the boundary conditions expressing the absence of the stresses at the surface. These boundary equations can be written in correspondence with those for the solid [9], but it is necessary to take into account the effect of the surface tension and the possibility of surface viscosity [10]. The general contributions of these two effects to the surface stresses are of the form. [10]

$$
\sigma_{z z}=-\gamma_{0} \frac{\mathrm{d}^{2} u_{3}}{\mathrm{~d} x_{1}^{2}}=\gamma_{0} k_{\|}^{2} u_{3},
$$

$$
\begin{aligned}
\sigma_{x z} & =-\left[\varepsilon-i \omega\left(\mu_{\mathrm{s}}+\mu_{\mathrm{s}}^{\prime}\right)\right] \frac{\mathrm{d}^{2} u_{1}}{\mathrm{~d} x_{1}^{2}} \\
& =k_{\|}^{2}\left[\varepsilon-i \omega\left(\mu_{\mathrm{s}}+\mu_{\mathrm{s}}^{\prime}\right)\right] u_{1},
\end{aligned}
$$

where $\gamma_{0}$ is the surface tension of the unstretched surface, $\varepsilon=\frac{\partial \gamma}{\partial(\log S)}$ (where $S$ is the liquid surface area) give the variation of the surface tension under stretching, and $\mu_{\mathrm{s}}, \mu_{\mathrm{s}}^{\prime}$ take account of the surface viscosity. Using the notation

$$
\bar{\varepsilon}=\varepsilon-i \omega\left(\mu_{\mathrm{s}}+\mu_{\mathrm{s}}^{\prime}\right),
$$

the boundary conditions at the free surface can be written

$$
\left[\left(i \omega \mu \frac{\mathrm{d}}{\mathrm{d} x_{3}}+k_{\|}^{2} \bar{\varepsilon}\right) g_{1 \alpha}-\omega \mu k_{\|} g_{3 \alpha}\right]_{x_{3}=0}=0,
$$




$$
\left[-i k_{\|}\left[\lambda-i \omega\left(\mu^{\prime}-\frac{2}{3} \mu\right)\right] g_{1 \alpha}+\left\{\left[-\lambda+i \omega\left(\mu^{\prime}+\frac{4}{3} \mu\right)\right] \frac{\mathrm{d}}{\mathrm{d} x_{3}}+\gamma_{0} k_{\|}^{2}\right\} g_{3 \alpha}\right]_{x_{3}=0}=0,
$$

$(\alpha=1,3)$

$$
\left[i \omega \mu \frac{\mathrm{d} g_{22}}{\mathrm{~d} x_{3}}\right]_{x_{3}=0}=0
$$

Let us define, as for the solid, the quantities

$$
\alpha_{\mathrm{t}}=\left(k_{\|}^{2}-\frac{\omega^{2}}{C_{\mathrm{t}}^{2}}\right)^{1 / 2}, \quad \alpha_{1}=\left(k_{\|}^{2}-\frac{\omega^{2}}{C_{1}^{2}}\right)^{1 / 2},
$$

and separate in the Green's function the bulk term $g^{(0)}$ from the surface contribution $g^{(S)}$

$$
g=g^{(0)}+g^{(S)} .
$$

The solution for $g^{(S)}$ can be written in the following form [9]

$$
\begin{aligned}
& g_{11}^{(S)}=A_{\mathrm{t}} \mathrm{e}^{-\alpha_{\mathrm{t}} x_{3}}+A_{1} \mathrm{e}^{-\alpha_{1} x_{3}}, \\
& g_{31}^{(S)}=\frac{i k_{\|}}{\alpha_{\mathrm{t}}} A_{\mathrm{t}} \mathrm{e}^{-\alpha_{\mathrm{t}} x_{3}}+\frac{i \alpha_{1}}{k_{\|}} A_{1} \mathrm{e}^{-\alpha_{1} \alpha_{3}}, \\
& g_{33}^{(S)}=A_{\mathrm{t}}^{\prime} \mathrm{e}^{-\alpha_{\mathrm{t}} x_{3}}+A_{1}^{\prime} \mathrm{e}^{-\alpha_{1} x_{3}}, \\
& g_{13}^{(S)}=-\frac{i \alpha_{\mathrm{t}}}{k_{\|}} A_{\mathrm{t}}^{\prime} \mathrm{e}^{-\alpha_{\mathrm{t}} x_{3}}-\frac{i k_{\|}}{\alpha_{1}} A_{1}^{\prime} \mathrm{e}^{-\alpha_{1} x_{3}}, \\
& g_{22}^{(S)}=B \mathrm{e}^{-\alpha_{\mathrm{t}} x_{3}} .
\end{aligned}
$$

Insertion of these solutions into the boundary conditions (10) yields the unknown coefficients $A_{\mathfrak{t}}, A_{\mathfrak{l}}, A_{\mathfrak{t}}^{\prime}, A_{\mathfrak{l}}^{\prime}$, $B$. The full expressions for these coefficients are given in the Appendix.

Let us just mention that the frequencies of the surface waves are given by the poles of this Green's function, and are then solutions of the equation

$$
\begin{gathered}
\left(\frac{k_{\|}^{2}+\alpha_{\mathrm{t}}^{2}}{\alpha_{\mathrm{t}}}+\frac{k_{\|}^{2} \vec{\varepsilon}}{\rho C_{\mathrm{t}}^{2}}\right)\left(2 C_{\mathrm{t}}^{2}-\frac{\omega^{2}}{k_{\|}^{2}}+\frac{\alpha_{1} \gamma_{0}}{\rho}\right)- \\
-\left(2 C_{\mathrm{t}}^{2}+\frac{\gamma_{0} k_{\|}^{2}}{\rho \alpha_{\mathrm{t}}}\right)\left(2 \alpha_{1}+\frac{k_{\|}^{2} \bar{\varepsilon}}{\rho C_{\mathrm{t}}^{2}}\right)=0 .
\end{gathered}
$$

In the simpler case of a non viscous liquid with $\varepsilon=0$, we obtain the usual capillary wave solution

$$
\rho \omega^{2}=\gamma_{0} k_{\|}^{2} \alpha_{1} .
$$

3. Surface density of states and specific heat of an inviscid liquid. - The above Green's function will be used in this section to study the surface contribution to the density of states and then to the low temperature specific heat of a liquid. We limit ourselves to a simpler case than that of section 2 by assuming $\varepsilon=0$ and by neglecting viscosity, but by keeping the surface tension $\gamma_{0}$ and the liquid compressibility.

The variation due to the surface of the bulk density of states follows from the imaginary part of the trace of the surface contribution to the Green's function
$\Delta n\left(\omega^{2}, k_{\|}\right)=-\frac{1}{\pi} \Im \mathrm{m} \int_{0}^{\infty}\left(g_{11}^{(S)}+g_{22}^{(S)}+g_{33}^{(S)}\right)_{x_{3}=x_{3}} \mathrm{~d} x_{3}$.

Since the dependence of the Green's function elements on $x_{3}$ and $x_{3}^{\prime}$ is of the form $\mathrm{e}^{-\left(\alpha_{i} x_{3}+\alpha_{j} x_{3}\right)}$, where $i, j=\mathrm{t}$ or 1 (see the Appendix), the integration in (15) is straightforward. Then we go to the limit of $\varepsilon=0$ and zero viscosity which leads to the following simple result

$$
\begin{aligned}
\Delta n\left(\omega^{2}, k_{\|}\right) & =-\frac{1}{\pi} \Im \mathrm{m}\left\{\frac{1}{4\left(\omega^{2}-C_{1}^{2} k_{\|}^{2}\right)}+\right. \\
& \left.+\frac{\omega^{2}-2 C_{1}^{2} k_{\|}^{2}}{2\left(\omega^{2}-C_{1}^{2} k_{\|}^{2}\right)\left(\omega^{2}-\frac{\gamma_{0} \alpha_{1} k_{\|}^{2}}{\rho}\right)}\right\} .
\end{aligned}
$$

We have to point out that on taking the imaginary part in equation (16), the quantity $\omega^{2}$ should be replaced by $\omega^{2}+i \eta$ where $\eta$ is an infinitesimal positive number. We then get three different contributions to $\Delta n$

$$
\Delta n=\Delta n^{(1)}+\Delta n^{(2)}+\Delta n^{(3)}
$$

$\Delta n^{(1)}$ results from the first term in (16)

$$
\Delta n^{(1)}=-\frac{1}{4} \delta\left(\omega^{2}-C_{1}^{2} k_{\|}^{2}\right) .
$$

It represents a discontinuity in the density of states at the threshold of the bulk band $\omega=C_{1} k_{\|}$; a similar result was obtained [11] at the longitudinal threshold in isotropic solids.

The second term in equation (16) gives rise to the two other contributions to $\Delta n$. The first of these contributions is

$$
\Delta n^{(2)}=\delta\left[\omega^{2}-\omega_{0}^{2}\left(k_{\|}\right)\right],
$$

where $\omega_{0}\left(k_{\|}\right)$is the frequency of the surface capillary wave, the solution of the equation (14b), that appears as a pole of the second term in equation (16). Let us point out that rearranging equation $(14 b)$ by means of the definition of $\alpha_{1}$ (Eq. (11)) leads to the following third order polynomial in $k_{\|}^{2}$

$$
k_{\|}^{6}-\frac{\omega_{0}^{2}}{C_{1}^{2}} k_{\|}^{4}-\frac{\rho^{2} \omega_{0}^{4}}{\gamma_{0}^{2}}=0 .
$$

Standard resolution of this equation shows [12] that it has only one real root

$$
\begin{aligned}
k_{\|}^{2}=\left[r+\left(q^{3}\right.\right. & \left.\left.+r^{2}\right)^{1 / 2}\right]^{1 / 3}+ \\
& +\left[r-\left(q^{3}+r^{2}\right)^{1 / 2}\right]^{1 / 3}+\frac{1}{3} \frac{\omega_{0}^{2}}{C_{1}^{2}},
\end{aligned}
$$




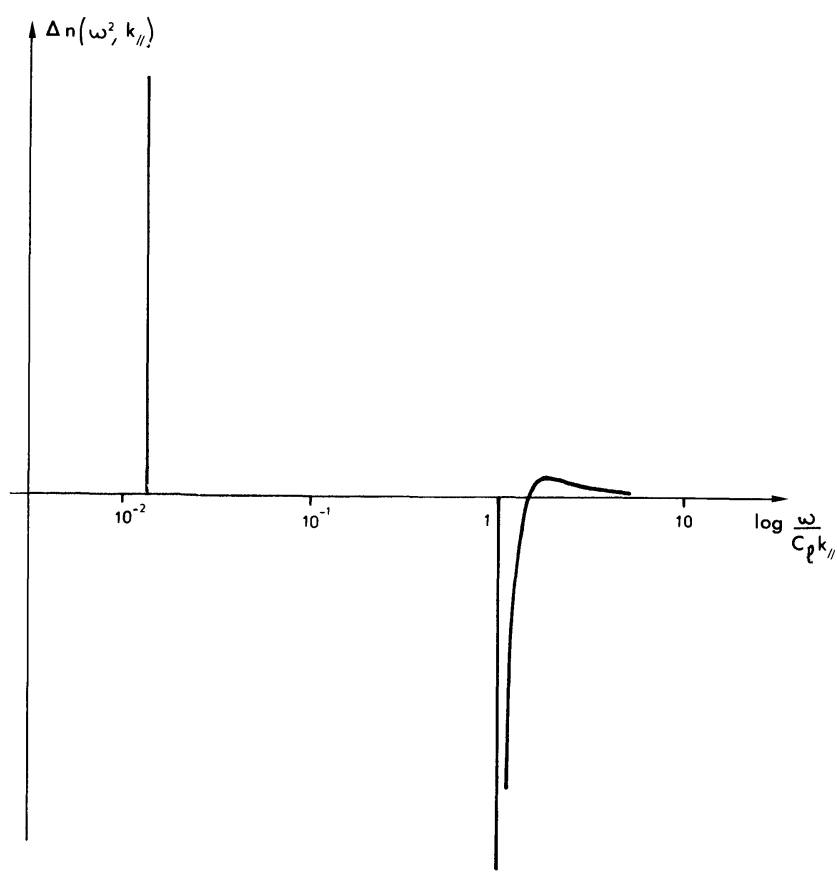

Fig. 1. - Surface density of states (in arbitrary units) for a given wavevector $k_{\|}$. The two vertical straight lines correspond to two delta functions of weights 1 and $-\frac{1}{4}$ respectively. The first one gives the position of the surface capillary wave, the second one is situated at the threshold of the bulk band. The figure is drawn for the parameters corresponding to liquid mercury (neglecting viscosity) and for $k_{\|}=10^{7} \mathrm{~m}^{-1}$. with

$$
r=\frac{\rho^{2} \omega_{0}^{4}}{2 \gamma_{0}^{2}}+\frac{1}{27}\left(\frac{\omega_{0}^{2}}{C_{1}^{2}}\right)^{3} \text { and } q=-\frac{1}{9}\left(\frac{\omega_{0}^{2}}{C_{1}^{2}}\right)^{2}
$$

For a given $k_{\|}$the frequency $\omega_{0}$ of this surface mode is below the threshold of the bulk band $C_{1} k_{\|}$.

Finally there is a variation of the density of states inside the bulk band that for $\omega>C_{1} k_{\|}$is

$$
\begin{aligned}
& \Delta n^{(3)}=\frac{-1}{2 \pi} \frac{\rho k_{\|}^{2}}{\gamma_{0}} \times \\
& \times \frac{\left(\frac{\omega^{2}}{C_{1}^{2}}-2 k_{\|}^{2}\right)}{\left(\frac{\omega^{2}}{C_{1}^{2}}-k_{\|}^{2}\right)^{1 / 2}\left(k_{\|}^{6}-\frac{\omega^{2}}{C_{1}^{2}} k_{\|}^{4}-\frac{\rho^{2} \omega^{4}}{\gamma_{0}^{2}}\right)} .
\end{aligned}
$$

An example of this density of states is given in figure 1. Now, we can evaluate the change in the specific heat due to the surface, calculating separately the contribution of each part of the density of states

$$
\Delta C_{V}=\Delta C_{V}^{(1)}+\Delta C_{V}^{(2)}+\Delta C_{V}^{(3)} .
$$

Let us recall that the surface contribution to the free energy is given by

$$
\Delta F(T)=\int_{0}^{\infty} \mathrm{d} \omega\left[\frac{\hbar \omega}{2}+k_{\mathrm{B}} T \log \left(1-\exp \left[-\frac{\hbar \omega}{k_{\mathrm{B}} T}\right]\right)\right] \int_{0}^{\infty} \frac{S}{(2 \pi)^{2}} \mathrm{~d}^{2} k_{\|} \Delta n\left(\omega, k_{\|}\right)
$$

where $S$ is the surface area, and $\Delta n\left(\omega, k_{\|}\right)=2 \omega \Delta n\left(\omega^{2}, k_{\|}\right)$.

Then, by using the relation

$$
\Delta C_{V}(T)=-T\left(\frac{\partial^{2} F}{\partial T^{2}}\right)_{V}
$$

we obtain the following formal expression for the surface specific heat

$$
\Delta C_{V}(T)=k_{\mathrm{B}} \int_{0}^{\infty}\left(\frac{\hbar \omega}{k_{\mathrm{B}} T}\right)^{2} \frac{\mathrm{d} \omega}{\left(\exp \left[\frac{\hbar \omega}{k_{\mathrm{B}} T}\right]-1\right)^{2}} \int \frac{S}{(2 \pi)^{2}} \mathrm{~d}^{2} k_{\|} 2 \omega \Delta n\left(\omega^{2}, k_{\|}\right) .
$$

The contribution of $\Delta n^{(1)}$ to the specific heat results directly from equations (27) and (18)

$$
\Delta C_{V}^{(1)}=-\frac{3 S k_{\mathrm{B}}}{8 \pi C_{1}^{2}}\left(\frac{k_{\mathrm{B}} T}{\hbar}\right)^{2} I(3)
$$

where we have defined

$$
I(u)=\int_{0}^{\infty} \frac{x^{u-1} \mathrm{~d} x}{\mathrm{e}^{x}-1}=\Gamma(u) \zeta(u)
$$

where $\zeta(u)$ is the Riemann zeta function. 
The second contribution to $\Delta C_{V}(T)$ comes from $\Delta n^{(2)}$, that is from the surface capillary wave

$$
\Delta C_{V}^{(2)}=\frac{S}{2 \pi} k_{\mathrm{B}} \int_{0}^{\infty} \frac{\left(\frac{\hbar \omega_{0}}{k_{\mathrm{B}} T}\right)^{2} \exp \left[\frac{\hbar \omega_{0}}{k_{\mathrm{B}} T}\right]}{\left(\exp \left[\frac{\hbar \omega_{0}}{k_{\mathrm{B}} T}\right]-1\right)^{2}} k_{\|} \mathrm{d} k_{\|} .
$$

In the equation (30) $\omega_{0}$ is a function of $k_{\|}$through equations (20)-(22), which give the dispersion of the surface wave.
Since the low temperature specific heat is governed by the low energy excitations of the system, we develop equation (21) in powers of $\omega_{0}$ to obtain

$$
k_{\|}^{2}=\left(\frac{\rho \omega_{0}^{2}}{\gamma_{0}}\right)^{2 / 3}+\frac{1}{3} \frac{\omega_{0}^{2}}{C_{1}^{2}}+\frac{1}{9}\left(\frac{\gamma_{0}}{\rho C_{1}^{6}}\right)^{2 / 3} \omega_{0}^{8 / 3}+\mathrm{O}\left(\omega_{0}^{10 / 3}\right) .
$$

Inserting this result into equation (30) one finds (with the aid of the relation $k_{\|} \mathrm{d} k_{\|}=\frac{1}{2} \mathrm{~d}\left(k_{\|}^{2}\right)$ )

$$
\Delta C_{V}^{(2)}=\frac{S k_{\mathrm{B}}}{\pi}\left\{\frac{7}{9}\left(\frac{\rho}{\gamma_{0}}\right)^{2 / 3}\left(\frac{k_{\mathrm{B}} T}{\hbar}\right)^{4 / 3} I(7 / 3)+\frac{1}{2 C_{1}^{2}}\left(\frac{k_{\mathrm{B}} T}{\hbar}\right)^{2} I(3)+\frac{22}{81}\left(\frac{\gamma_{0}}{\rho C_{1}^{6}}\right)^{2 / 3}\left(\frac{k_{\mathrm{B}} T}{\hbar}\right)^{8 / 3} I(11 / 3)\right\}+\mathrm{O}\left(T^{10 / 3}\right) .
$$

The term $T^{4 / 3}$ in (32) is the one obtained previously [2] for the contribution of the capillary wave to the specific heat while further corrections are of the form $T^{2}, T^{8 / 3}, T^{10 / 3}, T^{14 / 3}, T^{16 / 3}, \ldots$ Let us note that the $\Delta C_{V}^{(1)}$ term is partly compensated by the $T^{2}$ term appearing in $\Delta C_{V}^{(2)}$.

The last contribution to $\Delta C_{V}(T)$ results from $\Delta n^{(3)}$. In the denominator of $\Delta n^{(3)}$ (Eq. (23)), let us rewrite the term $k_{\|}^{6}-\frac{\omega^{2}}{C_{1}^{2}} k_{\|}^{4}-\frac{\rho^{2} \omega^{4}}{\gamma_{0}^{2}}$ in the new form

$$
\frac{\rho^{2} \omega^{4}}{\gamma_{0}^{2}}\left[1+\frac{\gamma_{0}^{2}}{\rho^{2}} k_{\|}^{4}\left(\frac{\omega^{2}}{C_{1}^{2}}-k_{\|}^{2}\right)\right] \text {, }
$$

pointing out that the region of interest for $k_{\|}$is $0<k_{\|}<\omega / C_{1}$. As the low lying excitations are the most important for our calculation, we can keep in the preceding expression the $\rho^{2} \omega^{4} / \gamma_{0}^{2}$ as the leading part, the remainder being considered in a Taylor series expansion

$$
\begin{aligned}
\frac{1}{\frac{\rho^{2} \omega^{4}}{\gamma_{0}^{2}}\left[1+\frac{\gamma_{0}^{2} k_{\|}^{4}}{\rho^{2}}\left(\frac{\omega^{2}}{C_{1}^{2}}-k_{\|}^{2}\right)\right]} & \simeq \\
& \simeq \frac{1}{\frac{\rho^{2} \omega^{4}}{\gamma_{0}^{2}}}\left[1-\frac{\gamma_{0}^{2} k_{\|}^{4}}{\rho^{2}}\left(\frac{\omega^{2}}{C_{1}^{2}}-k_{\|}^{2}\right)+\cdots\right] .
\end{aligned}
$$

Then we get immediately

$$
\Delta C_{V}^{(3)}=\frac{-4 S k_{\mathrm{B}}}{5 \pi^{2}}\left(\frac{k_{\mathrm{B}} T}{\hbar}\right)^{3} \frac{\gamma_{0}}{\rho C_{1}^{5}} I(4)+\mathrm{O}\left(T^{5}\right) .
$$

Finally the surface specific heat of a non viscous fluid can be obtained by adding the contributions of equations (28), (32) and (33). Let us write it in the following form

$$
\begin{aligned}
\Delta C_{V}(T) & =S \frac{k_{\mathrm{B}}}{\pi}\left[M\left(\frac{T}{T_{0}}\right)^{4 / 3}+N\left(\frac{T}{T_{0}}\right)^{2}+\right. \\
& \left.+P\left(\frac{T}{T_{0}}\right)^{8 / 3}+Q\left(\frac{T}{T_{0}}\right)^{3}+\mathrm{O}\left(T^{10 / 3}\right)\right],
\end{aligned}
$$

where the coefficients are

$$
\begin{aligned}
& M=\frac{7}{9}\left(\frac{\rho}{\gamma_{0}}\right)^{2 / 3}\left(\frac{k_{\mathrm{B}} T_{0}}{\hbar}\right)^{4 / 3} I\left(\frac{7}{3}\right), \\
& P=\frac{22}{81}\left(\frac{\gamma_{0}}{\rho C_{1}^{6}}\right)^{2 / 3}\left(\frac{k_{\mathrm{B}} T_{0}}{\hbar}\right)^{8 / 3} I\left(\frac{11}{3}\right), \\
& N=\frac{1}{8 C_{1}^{2}}\left(\frac{k_{\mathrm{B}} T_{0}}{\hbar}\right)^{2} I(3), \\
& Q=\frac{-4}{5 \pi} \frac{\gamma_{0}}{\rho C_{1}^{5}}\left(\frac{k_{\mathrm{B}} T_{0}}{\hbar}\right)^{3} I(4) .
\end{aligned}
$$

The function $I(u)$ was defined by equation (29) and the arbitrary temperature $T_{0}$ was introduced just in order to get for $M, P, N$ and $Q$ quantities with the same dimension $\left(\mathrm{m}^{-2}\right)$. The contribution in $T^{4 / 3}$ is the same as the one predicted by Atkins [2]. Iosilevskii's [3] result for the specific heat of a free liquid surface is the same as our contribution $\Delta C_{V}^{(1)}$ (Eq. (28)) coming from the threshold at $C_{1}$. He did not take into account any localized surface mode below the bulk bands and obtained finally that the creation of a free surface decreases the density of vibrational states. In principle the creation of a free surface produces the opposite effect, as in our present calculation.

The last term in equation (34) is of the same order in $T$ as the contribution of the bulk waves to the specific heat, that is [13]

$$
C_{V}^{(\mathrm{B})}(T)=\frac{2 \pi^{2}}{15} V k_{\mathrm{B}}\left(\frac{k_{\mathrm{B}} T}{\hbar}\right)^{3} \frac{1}{C_{1}^{3}},
$$

where $V$ is the volume of the liquid. 
Let us illustrate the above calculation by the example of liquid helium, comparing the magnitude of the different coefficients $M, N, P, Q$. For the liquid ${ }^{4} \mathrm{He}$, the velocity of sound and the density are [3]

$$
C_{1}=238 \mathrm{~m} / \mathrm{s} \text { and } \rho=145 \mathrm{~kg} / \mathrm{m}^{3}
$$

and the surface tension is [14] $\gamma_{0}=3.5 \times 10^{-4} \mathrm{~J} \cdot \mathrm{m}^{-2}$. Taking $T_{0}=1 \mathrm{~K}$ one finds in units of $10^{17} \mathrm{~m}^{-2}$

$$
M \simeq 48.2, \quad N \simeq 0.9, \quad P \simeq 0.3, \quad Q \simeq-0.1 .
$$

We can also compare the $\Delta C_{V}^{(3)}$ contribution, which behaves like $T^{3}$, to the bulk specific heat

$$
\left|\frac{\Delta C_{V}^{(3)}}{C_{V}^{(\mathrm{B})}}\right|=\frac{6}{\pi^{4}} \frac{\gamma_{0}}{\rho C_{1}^{2}} \frac{S}{V} \simeq\left(2.6 \times 10^{-12} \mathrm{~m}\right) \times \frac{S}{V} .
$$

The above quantities enable us to find for liquid helium the relative importance of the surface specific heat for a given ratio of the surface to the volume.

Finally, a different example is given by liquid mercury for which [7]

$$
\begin{gathered}
\gamma_{0}=0.55 \mathrm{~J} . \mathrm{m}^{-2}, \quad \rho=13.6 \times 10^{3} \mathrm{~kg} / \mathrm{m}^{3}, \\
C_{1}=1500 \mathrm{~m} / \mathrm{s} .
\end{gathered}
$$

It is more convenient to take $T_{0}=300 \mathrm{~K}$ in this case. Then in units of $10^{20} \mathrm{~m}^{-2} M \simeq 14.8, N \simeq 2, P \simeq 5$, $Q \simeq-5.3$ and

$$
\left|\frac{\Delta C_{V}^{(3)}}{C_{V}^{(\mathrm{B})}}\right| \simeq\left(1.1 \times 10^{-12} \mathrm{~m}\right) \frac{S}{V} .
$$

The higher terms play a more important role here than in the case of liquid helium.

4. Discussion. - We present in this section a summary and discussion of the results obtained above.

The Green's function of a liquid with a free surface has been calculated taking into account the surface tension and the viscosity. Various forms of this Green's function were recently used to discuss the intensity of the light scattered from a liquid surface [6-8]. The expressions derived here are shown to be formally identical with those for an isotropic solid. Therefore, they put the solid and the liquid on the same mathematical footing. Also, they can easily be generalized to describe interfaces between liquids or between solids and liquids.

The contribution of the surface to the density of states and to the specific heat are then derived for an inviscid fluid. For a given $\mathbf{k}_{\|}$the density of states is composed of three components : a delta function (of weight one) at the frequency $\omega_{\mathrm{c}}$ of the surface capillary wave, another delta function (of weight $-\frac{1}{4}$ ) yielding a loss of states at the threshold of the bulk band, and finally a continuous variation inside the bulk band. Adding the viscosity to the problem enlar- ges the two delta peaks which are the more important component of the surface density of states. In particular, at the limit of low viscosity, the delta function corresponding to the capillary wave yields a lorentzian centred at $\omega_{\mathrm{c}}$ of width $\Delta \omega \simeq \frac{2 \mu k_{\|}^{2}}{\rho}$; for typical values of $k_{\|}$in scattering experiment, let us say $10^{5}-10^{7} \mathrm{~m}^{-1}$, $\omega_{\mathrm{c}}$ is given to a good approximation by $\omega_{\mathrm{c}} \simeq \frac{\gamma_{0} k_{\|}^{3 / 2}}{\rho}$, i.e. the result for an incompressible fluid $(C \rightarrow \infty)$. As the relative width $\Delta \omega / \omega_{c}$ of this lorentzian is an increasing function of $k_{\|,}$, the viscosity plays a more important role for higher wavevectors.

The surface specific heat of an inviscid fluid $\Delta C_{V}$ was calculated as a series expansion in the temperature $T$ up through the term in $T^{3}$. The capillary wave gives the main contribution to $\Delta C_{V}$ which is in $T^{4 / 3}$ and then correction terms of type $T^{2}, T^{8 / 3}, T^{10 / 3}$, etc... The term in $T^{2}$ is partially compensated by the contribution of the delta peak in the density of states situated at the bulk threshold. Finally the variation of the density of states inside the bulk band only contributes to $\Delta C_{V}$ by a term in $T^{3}$ which has the same power dependence as the bulk specific heat.

Obviously $\Delta C_{V}$ as a whole is proportional to the surface area while the bulk specific heat is proportional to the volume. The examples given above show that even for droplets of micron size the determination of $\Delta C_{V}$ needs good precision (about $10^{-4}$ ) as well as low temperatures. Finally we should stress that for very small droplets (i) the surface area is not planar, (ii) the wavevectors are limited by a minimum cut-off of the order of the inverse of the droplet radius, and (iii) the viscosity may play a role at these wavevectors. Then further studies of the properties reported here including viscosity and different geometries are needed.

Acknowledgments. - We would like to thank Professors A. A. Maradudin and R. F. Wallis and Dr. V. Velasco for their interest in this work.

\section{APPENDIX}

Expression of the Green's function for the semiinfinite liquid. - We do not give here the bulk Green's function of the liquid which have exactly the same expression as for an isotropic solid [9], once the values of $C_{1}$ and $C_{\mathrm{t}}$ given by equation (6) are used. The coefficients $A_{\mathfrak{t}}, A_{1}, A_{\mathfrak{t}}^{\prime}, A_{1}^{\prime}, B$ appearing in the surface part (Eqs. (12)) are given by

$A_{\mathrm{t}}=-\frac{k_{\|}^{2}}{\omega^{2} \Delta}\left\{\left[2\left(2 C_{\mathrm{t}}^{2}-\frac{\omega^{2}}{k_{\|}^{2}}\right)-\frac{k_{\|}^{2} \bar{\varepsilon}}{\rho C_{\mathrm{t}}^{2}} \frac{\gamma_{0}}{\rho}\right] \mathrm{e}^{-\alpha_{1} x_{3}^{\prime}}+\right.$ 


$$
\begin{aligned}
& \left.+\frac{C_{\mathrm{t}}^{2}}{2} \mathrm{e}^{-\alpha_{\mathrm{t}} x^{\prime}}\left[-\frac{4 \alpha_{\mathrm{t}} \alpha_{1}}{k_{\|}^{2}}-\left(2-\frac{\omega^{2}}{C_{\mathrm{t}}^{2} k_{\|}^{2}}\right)^{2}+\frac{\gamma_{0} \alpha_{1}}{\rho C_{\mathrm{t}}^{2}} \frac{\omega^{2}}{C_{\mathrm{t}}^{2} k_{\|}^{2}}-\frac{\alpha_{\mathrm{t}} \bar{\varepsilon}}{\rho C_{\mathrm{t}}^{2}} \frac{\omega^{2}}{C_{\mathrm{t}}^{2} k_{\|}^{2}}+\frac{\gamma_{0}}{\rho C_{\mathrm{t}}^{2}} \frac{\bar{\varepsilon}}{\rho C_{\mathrm{t}}^{2}}\left(\alpha_{1} \alpha_{\mathrm{t}}+k_{\|}^{2}\right)\right]\right\}, \\
& A_{1}=-\frac{k_{\|}^{2}}{2 \omega^{2} \Delta}\left\{\left[\frac{k_{\|}^{2}+\alpha_{\mathrm{t}}^{2}}{\alpha_{\mathrm{t}} \alpha_{1}}\left(\frac{\omega^{2}}{k_{\|}^{2}}-2 C_{\mathrm{t}}^{2}\right)-4 C_{\mathrm{t}}^{2}-\frac{\gamma_{0}}{\rho \alpha_{\mathrm{t}}} \frac{\omega^{2}}{C_{\mathrm{t}}^{2}}+\frac{k_{\|}^{2} \bar{\varepsilon}}{\rho \alpha_{1}} \frac{\omega^{2}}{C_{\mathrm{t}}^{2} k_{\|}^{2}}+\right.\right. \\
& \left.\left.+\frac{\bar{\varepsilon} \gamma_{0} k_{\|}^{2}}{\rho^{2} C_{\mathrm{t}}^{2}}\left(1+\frac{k_{\|}^{2}}{\alpha_{\mathrm{t}} \alpha_{1}}\right)\right] \mathrm{e}^{-\alpha_{1} x_{3}^{\prime}}+\left[4 C_{\mathrm{t}}^{2} \frac{k_{\|}^{2}+\alpha_{\mathrm{t}}^{2}}{k_{\|}^{2}}-2 \frac{\gamma_{0} \bar{\varepsilon} k_{\|}^{2}}{\rho^{2} C_{\mathrm{t}}^{2}}\right] \mathrm{e}^{-\alpha_{\mathrm{t}} x_{3}^{\prime}}\right\} \\
& A_{1}^{\prime}=\frac{\alpha_{1}}{\alpha_{\mathrm{t}}} \frac{k_{\|}^{2}}{2 \omega^{2} \Delta}\left\{\mathrm { e } ^ { - \alpha _ { 1 } x _ { 3 } } \left[C_{\mathrm{t}}^{2} \frac{\left(k_{\|}^{2}+\alpha_{\mathrm{t}}^{2}\right)^{2}+4 \alpha_{\mathrm{t}} \alpha_{1} k_{\|}^{2}}{k_{\|}^{4}}+\frac{\gamma_{0} \omega^{2} \alpha_{1}}{\rho C_{\mathrm{t}}^{2} k_{\|}^{2}}-\frac{\bar{\varepsilon} \omega^{2} \alpha_{\mathrm{t}}}{\rho C_{\mathrm{t}}^{2} k_{\|}^{2}}-\right.\right. \\
& \left.\left.-\frac{\gamma_{0} \bar{\varepsilon}}{\rho^{2} C_{\mathrm{t}}^{2}}\left(k_{\|}^{2}+\alpha_{\mathrm{t}} \alpha_{1}\right)\right]-\mathrm{e}^{-\alpha_{\mathrm{t}} x_{3}^{\prime}}\left[4 C_{\mathrm{t}}^{2} \frac{k_{\|}^{2}+\alpha_{\mathrm{t}}^{2}}{k_{\|}^{2}}-2 \frac{\gamma_{0} \bar{\varepsilon} k_{\|}^{2}}{\rho^{2} C_{\mathrm{t}}^{2}}\right]\right\} \\
& A_{\mathrm{t}}^{\prime}=-\frac{\alpha_{1}}{\alpha_{\mathrm{t}}} \frac{k_{\|}^{2}}{2 \omega^{2} \Delta}\left\{\mathrm{e}^{-\alpha_{1} x_{3}^{\prime}}\left[\frac{4 C_{\mathrm{t}}^{2}\left(k_{\|}^{2}+\alpha_{\mathrm{t}}^{2}\right)}{k_{\|}^{2}}-\frac{2 \gamma_{0} \bar{\varepsilon} k_{\|}^{2}}{\rho^{2} C_{\mathrm{t}}^{2}}\right]+\right. \\
& \left.+\mathrm{e}^{-\alpha_{\mathrm{t}} x_{3}^{2}}\left[-C_{\mathrm{t}}^{2} \frac{\left(k_{\|}^{2}+\alpha_{\mathrm{t}}^{2}\right)^{2}+4 \alpha_{\mathrm{t}} \alpha_{1} k_{\|}^{2}}{\alpha_{\mathrm{t}} \alpha_{1}}+\frac{\gamma_{0} \omega^{2}}{\rho \alpha_{\mathrm{t}} C_{\mathrm{t}}^{2}}-\frac{\bar{\varepsilon} \omega^{2}}{\rho \alpha_{1} C_{\mathrm{t}}^{2}}+\frac{\gamma_{0} \bar{\varepsilon}}{\rho^{2} C_{\mathrm{t}}^{2}} \frac{k_{\|}^{2}+\alpha_{\mathrm{t}} \alpha_{1}}{\alpha_{\mathrm{t}} \alpha_{1}}\right]\right\},
\end{aligned}
$$

with

and

$$
\Delta=\left(\frac{k_{\|}^{2}+\alpha_{\mathrm{t}}^{2}}{\alpha_{\mathrm{t}}}+\frac{k_{\|}^{2} \bar{\varepsilon}}{\rho C_{\mathrm{t}}^{2}}\right)\left(2 C_{\mathrm{t}}^{2}-\frac{\omega^{2}}{k_{\|}^{2}}+\frac{\alpha_{1} \gamma_{0}}{\rho}\right)-\left(2 C_{\mathrm{t}}^{2}+\frac{\gamma_{0} k_{\|}^{2}}{\rho \alpha_{\mathrm{t}}}\right)\left(2 \alpha_{1}+\frac{k_{\|}^{2} \bar{\varepsilon}}{\rho C_{\mathrm{t}}^{2}}\right)
$$

$$
B=-\frac{1}{2 C_{\mathrm{t}}^{2} \alpha_{\mathrm{t}}} \text {. }
$$

\section{References}

[1] See for references, Maradudin, A. A., Wallis, R. F. and DoBrZYNSKI, L., in Surface phonons and polaritons, Handbook of Surfaces and Interfaces, Vol. 3, Ed. L. Dobrzynski (Garland Press, New York) 1980.

[2] Atkins, K. R., 1953 in Statistical Physics;

Landau, L. and Lifshitz, E. M., (Pergamon Press) 1959, p. 460.

[3] IosilevskiI, YA. A., Phys. Rev. B 20 (1979) 1473.

[4] Duafari-Rouhani, B., Thesis, Université Paris-Sud (1978).

[5] Garcia-Moliner, F., Ann. Phys. (Paris) 2 (1977) 179.

[6] Platero, G., Velasco, V. R. and Garcia-Moliner, F., Phys. Scr. 23 (1981) 1108.

[7] Loudon, R., Proc. R. Soc. London A 372 (1980) 275.
[8] Bouchiat, M. A. and Meunier, J., J. Physique Colloq. 33 (1972) C1-141.

Langevin, D. and Bouchiat, M. A., J. Physique 33 (1972) 101.

[9] Maradudin, A. A. and Mills, D. L., Ann. Phys. 100 (1976) 262.

[10] Hansen, R. A., Ahmad, J. A., Prog. Surf. Member Sci. 4 (1971) 1.

[11] BurT, M. G., J. Phys. C 6(1973) 855.

[12] Handbook of Mathematical functions, Eds. M. Abramowitz, I. A. Stegun (Dover Publications, I.N.C. New York) p. 17.

[13] See reference [2], p. 200.

[14] American Institute of Physics Handbook, 3rd Edition, Ed. D. E. Gray (Mc Graw Hill) 1972, p. 2-207. 